ARTICLE

https://doi.org/10.1038/s42004-019-0113-8

OPEN

\title{
Mechanochromism induced through the interplay between excimer reaction and excited state intramolecular proton transfer
}

\author{
Yu-Chen Wei ${ }^{1}$, Zhiyun Zhang ${ }^{1,2}$, Yi-An Chen ${ }^{1}$, Cheng-Ham Wu', Zong-Ying Liu', Ssu-Yu Ho ${ }^{1}$, Jiun-Chi Liu', \\ Jia-An $\operatorname{Lin}^{1} \&$ Pi-Tai Chou ${ }^{1}$
}

Excited-state intramolecular proton transfer (ESIPT) and excimer formation are unimolecular and bimolecular reactions, respectively. The coupling between these two reactions has been rarely observed. Here we show the interplay between ESIPT and excimer formation in $\mathbf{C F}_{\mathbf{3}}$-HTTH (2,2'-(thiazolo[5,4-d]thiazole-2,5-diyl)bis(4-(trifluoromethyl)phenol) solid crystal, resulting in the extensively broad multiple emissions spanning from 420 to $750 \mathrm{~nm}$. Comprehensive structural, time-resolved spectroscopic, and two-photon microscopic studies of $\mathbf{C F}_{\mathbf{3}}-\mathbf{H T T H}$ in crystal lattice unveil the pre-equilibrium ESIPT between the normal $\left(\mathrm{N}^{\star}\right)$ and tautomer $\left(T^{\star}\right)$ form, for which the slow population decay and well-ordered lattice packing facilitate excimeric $\left(E^{\star}\right)$ formation, rendering $N^{\star}(460 \mathrm{~nm}), T^{\star}(520 \mathrm{~nm})$ and $E^{\star}(600 \mathrm{~nm})$ triple emissions. In contrast, the lower degree of packing on the solid surface prohibits excimer formation, showing only the ESIPT process. The correlation between luminescence properties and packing structure sheds light on the corresponding mechanochromic effect based on molecular solid architecture and provide new insight into the aggregationinduced properties.

\footnotetext{
${ }^{1}$ Department of Chemistry, National Taiwan University, Taipei 10617 Taiwan, Republic of China. ${ }^{2}$ Key Laboratory for Advanced Materials and Institute of Fine Chemicals, East China University of Science \& Technology, Shanghai 200237, P.R. China. These authors contributed equally: Yu-Che Wei, Zhiyun Zhang. Correspondence and requests for materials should be addressed to Z.Z. (email: zhangzhiyun@ecust.edu.cn) or to P.-T.C. (email: chop@ntu.edu.tw)
} 
O rganic luminescence in solid state is an intense area of investigation owing to its versatile applications ${ }^{1-3}$. The broad interest in solid-state emissions has led to increased interest in high photoluminescence quantum yield (PLQY) at ultra-blue ${ }^{4-7}$, white-light ${ }^{8,9}$ and near-infrared region $^{10,11}$, temperature-dependent color changes for sensing ${ }^{12,13}$, mechanoluminescence and mechanochromism ${ }^{14,15}$, and micro-cavity laser ${ }^{16-18}$. To further tune these properties, understanding and hence manipulation of their light-emitting behavior in solid state is crucial, especially the alliance between structure of molecular solid and the corresponding excited-state properties. Some of the frequently reported properties include aggregation-induced emission (AIE) or aggregation-caused quenching (ACQ) to describe relevant phenomena ${ }^{8,9}$.

The excimer formation in solid state is the other key area of interest, which is defined as an electronically excited molecule interacting with the proximal congeners, forming a dimer-like configuration that otherwise has no bonding formation in the ground state ${ }^{19}$. Due to the repulsive ground-state potential energy surface, the emission features are unstructured and exhibits large Stokes shift as compared to the corresponding monomer ${ }^{20}$. Its dynamics incorporating four electronic levels has been extensively applied to laser action ${ }^{21}$. Empirically, organic excimer formed in solid state requires molecular stacking layer-by-layer in an interplanar distance around $3.5 \AA$ such that they are virtually in dimers- or oligomers- like arrangement ${ }^{22}$. Similarly, excited-state intramolecular proton transfer (ESIPT) reaction in solid state is another interesting property. ESIPT is the relocation of the proton in the excited state, giving rise to an isomer (proton-transfer tautomer) species that exhibits emission with anomalously large Stokes shift with respect to the absorption. In contrast to the above-mentioned bi- or aggregate-molecular event, ESIPT is a unimolecular reaction that is subjected to less environmental perturbation in solid. This unique property has led to numerous fundamental approaches ${ }^{23-28}$ and cutting-edge applications ${ }^{29-31}$. One of recent advances can be ascribed to the ESIPT coupled thermally activated delay fluorescence (TADF) system, rendering high performance OLED with external quantum efficiency (EQE) of around $14 \%{ }^{32}$.

Here we show the aggregation phenomena by reporting the simultaneous ESIPT coupled excimer formation in a system CF $_{3}$-HTTH $\quad\left(2,2^{\prime}\right.$-(thiazolo[5,4-d]thiazole-2,5-diyl)bis(4-(trifluoromethyl)phenol), Fig. 1a), rendering a triple emission spanning from 420 to $750 \mathrm{~nm}$ in a single-type molecular solid. Two-photon excitation microscopy, which is well-established in biological imaging but rarely applied in material emission microscopy in organic solid ${ }^{33,34}$, spatially (in depth) resolved the ratiometric emission that depends on degree of packing from the crystal surface to kernel. These, together with the studies of the molecules $\mathbf{C F}_{\mathbf{3}}$ - MTTH and $\mathbf{C F}_{\mathbf{3}}$-MTTM that possess one and none proton donating sites, respectively, as well as a series of other $\mathbf{C F}_{\mathbf{3}}$-HTTH congeners as reference compounds, lead us to unify structural criteria undergoing ESIPT coupled excimer reactions. Note that the system exhibiting ESIPT and excimer emissions has been reported. However, the compound contains two moieties that undergo excimer formation and AIE-ESIPT separately without correlation ${ }^{35}$. In sharp contrast, as evidenced by the time-resolved spectroscopy and dynamics of the emission, our results clearly shed light on the direct correlation between the ESIPT and excimer dynamic processes.

\section{Results}

Synthesis and crystal structures. Syntheses of compounds CF - -HTTH, CF 3 -MTTH (2-(5-(2-methoxy-5-(trifluoromethyl)<smiles>Oc1ccc(Cl)cc1-c1nc2sc(-c3cc(C(F)(F)F)ccc3O)nc2s1</smiles>

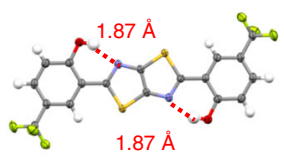

C

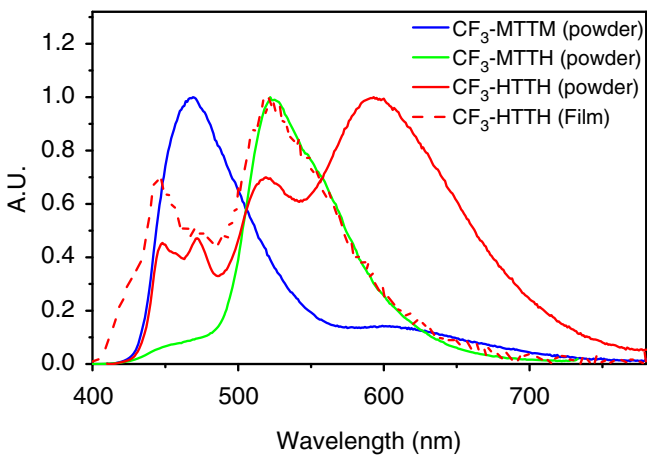

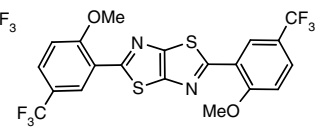
$\mathrm{CF}_{3}-\mathrm{MTTH}$

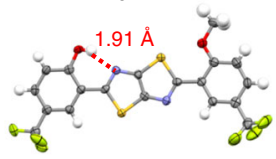

$\mathrm{CF}_{3}-\mathrm{MTTM}$

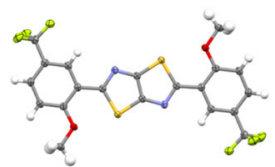

b

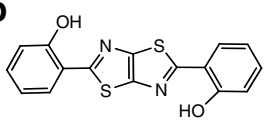

HTTH

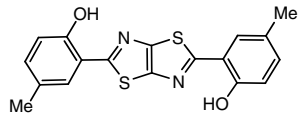

$m$-HTTH

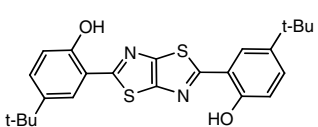

$t$-HTTH

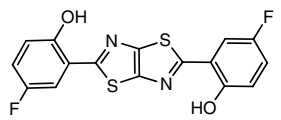

F-HTTH
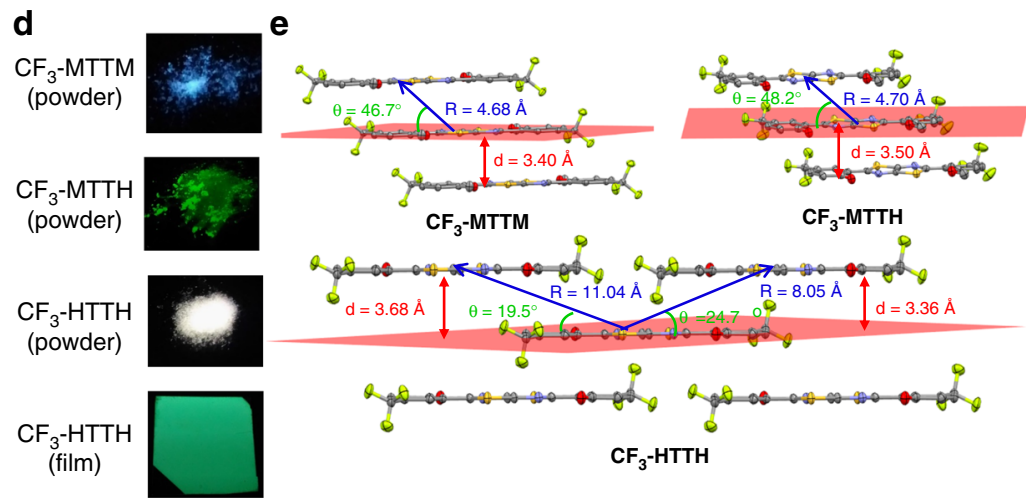

Fig. 1 Molecular and crystal structures. a The structures and ellipsoids at the 50\% probability level. Carbon, oxygen, sulfur, nitrogen, and fluorine atoms are shown as gray, red, yellow, blue and yellow-green, respectively. Hydrogen atoms are simulated by software and the red dotted lines show the hydrogen bond distance. Also indicated are the H-bond distances. b Molecular structures of reference compounds HTTH, $\mathbf{t}$-HTTH, $\boldsymbol{m}$-HTTH, and F-HTTH. c Room temperature steady-state emission of $\mathbf{C F}_{\mathbf{3}}-\mathbf{H} \mathbf{T} \mathbf{T H}, \mathbf{C F}_{\mathbf{3}}-\mathbf{M} \mathbf{M T H}$ and $\mathbf{C F}_{\mathbf{3}}-\mathbf{M T T M}$ in pristine solid powder (solid line). Also shown is $\mathbf{C F}_{\mathbf{3}}-\mathbf{H} \mathbf{T T H}$ in solid thin film (dashed line) prepared by vapor deposition on the quartz substrate. $\mathbf{d}$ The naked eyes view of the emission of three compounds in solid powder and $\mathbf{C F}_{\mathbf{3}}-\mathbf{H T T H}$ in solid thin film. The excitation wavelength is $380 \mathrm{~nm}$. e The Stacking images in the crystal of $\mathbf{C F}_{\mathbf{3}}-\mathbf{M} \mathbf{M} \mathbf{M}, \mathbf{C F} \mathbf{F}_{\mathbf{3}}-\mathbf{M T T H}$ and $\mathbf{C F}_{\mathbf{3}}-\mathbf{H T T H}$. Red vectors represent the interplanar distances $d$. $\theta$ is denoted by green angles. Blue vectors indicate the intermolecular distances $R$ 
Table 1 Comparison of crystal structures of HTTH series compounds and the tendency of excimer formation

\begin{tabular}{|c|c|c|c|c|}
\hline & Interplanar distance $d(\AA)^{a}$ & $\theta$ (degree) $^{\mathbf{b}}$ & Intermolecular distance $R(\AA) c$ & Excimer formation \\
\hline $\mathrm{CF}_{3}-\mathrm{MTTM}$ & 3.40 & 46.7 & 4.68 & $\checkmark$ \\
\hline $\mathrm{CF}_{3}-\mathrm{MTTH}$ & 3.50 & 48.2 & 4.70 & $x$ \\
\hline $\mathrm{CF}_{3}-\mathrm{HTTH}$ & $3.68,3.36$ & $19.5,24.7$ & $11.04,8.05$ & $\checkmark$ \\
\hline HTTH & 3.40 & 42.0 & 5.09 & $x$ \\
\hline$t$-HTTH & 3.48 & 35.2 & 6.04 & $x$ \\
\hline m-HTTH & 3.35 & 26.7 & 7.47 & $x$ \\
\hline F-HTTH & 3.37 & 86.5 & 3.77 & $x$ \\
\hline
\end{tabular}

phenyl)thiazolo[5,4-d]thiazol-2-yl)-4-(trifluoromethyl)phenol, Fig. 1a) and $\mathbf{C F}_{3}-\mathbf{M T T M}\left(2,2^{\prime}\right.$-(thiazolo[5,4-d]thiazole-2,5-diyl) bis(4-methylphenol), Fig. 1a) were according to a procedure described in our previous work on $\boldsymbol{t}$-HTTH $\left(2,2^{\prime}\right.$-(thiazolo[5,4-d] thiazole-2,5-diyl)bis(4-tert-butyl-phenol, Fig. 1b) ${ }^{36}$ with slight modification (Supplementary Figure 1). Their single crystals were all obtained by slow evaporation of solvent from the mixture solutions of dichloromethane/ethanol and examined by X-ray analysis (Supplementary Data 1-12). As shown in Fig. 1e, the molecular structures of the three titled compounds are all nearly planar. The dual and single intramolecular $\mathrm{H}$-bond formation in $\mathbf{C F}_{3}-\mathbf{H T T H}$ and $\mathrm{CF}_{3}-\mathbf{M T T H}$ crystal is apparent (Fig. 1a), which is also supported by the H-NMR with large down field shifted proton peaks at $\delta 12.30$ and $\delta 12.35 \mathrm{ppm}$, respectively, in DMSO$\mathrm{d}_{6}$ (Supplementary Figure 9 and 10). Despite the sameness in molecular structure, the existence of $-\mathrm{CF}_{3}$ and/or $-\mathrm{OCH}_{3}$ makes they much different in molecular packing, manifesting their difference in photophysics and excited-state reaction dynamics in solid (see below).

Steady-state behaviors of solid. The three congeners in solid, shown in Fig. 1c, d, exhibit much different photophysical property (Supplementary Table 1 ). For $\mathbf{C F}_{3}-\mathbf{H T T H}$ in pristine solid powder, in addition to dual emission bands located at around 440 $\mathrm{nm}$ (the first vibronic peak) and $520 \mathrm{~nm}$, a major red emission band appears at $600 \mathrm{~nm}$; the combination of these three emission bands shows prominent white light generation (Fig. 1c, red line). Upon monitoring at three individual emission regions, similar excitation spectra are obtained (Supplementary Figure 2), indicating that they all originate from the same ground-state species. Because spectral features and location of $440 \mathrm{~nm}$ and $520 \mathrm{~nm}$ bands are similar to those of the dual emission in solution (Supplementary Figure 3 and Supplementary Note 1), it is reasonable to attribute them to the normal and proton-transfer tautomer emissions, respectively (see below). This viewpoint is supported by the disappearance of tautomer $520 \mathrm{~nm}$ emission in solid $\mathbf{C F}_{3}$-MTTM (Fig. 1c, blue line) where ESIPT is prohibited due to the lack of proton donor (see Fig. 1a for structure). The $600 \mathrm{~nm}$ emission band is broad and lacks any prominent vibronic progression, which is not observed in solution and disappears when $\mathbf{C F}_{3}-\mathbf{H T T H}$ is deposited as a thin film on the quartz substrate via vapor deposition or dissolved in a PMMA film (Fig. 1c, dashed line and Supplementary Figure 4). Therefore, its origin associated with some sort of self-assembled intermolecular interaction is unambiguous. Accordingly, we tentatively assign the $600 \mathrm{~nm}$ emission band in the solid to the excimer emission. The coexistence of ESIPT and excimeric reactions in a single type molecular solid, to our knowledge, is unprecedented. As for the origin of excimer formation, on the one hand, ESIPT does not seem to be the prerequisite for the excimer formation. This viewpoint is supported by the observation of similar $600 \mathrm{~nm}$ excimer emission in $\mathbf{C F}_{3}$-MTTM solid, where no ESIPT takes place (Fig. 1c, blue line). Alternatively, the excimer formation should be related to the molecular packing in the solid, as supported by $\mathrm{CF}_{3}-\mathrm{MTTH}$ that exhibits dual emission (440 and $520 \mathrm{~nm}$ ) via ESIPT in solid but lacks excimer emission (Fig. 1c, green line).

To gain further insight into the molecular packing, based on the $\mathrm{x}$-ray structures, we then examine the layer-by-layer crystal structure of these compounds for possible interactions. According to Kasha's exciton model ${ }^{37}$, the coupling of transition dipole moment plays an important role in determining the Stokes shift of the emission spectrum in solid. Using the point-dipole approximation, the energy splitting of infinite linear polymer, $\Delta E$, induced by the alignment of transition dipole, can be expressed as ${ }^{38}$ :

$$
\Delta E_{\text {linear }} \sim \frac{2|\vec{\mu}|^{2}}{4 \pi \varepsilon R^{3}}\left(1-3 \cos ^{2} \theta\right)
$$

where $\vec{\mu}$ is the transition dipole of a monomer. $R$ is the intermolecular distance between center of mass of the chromophore. $\theta$ is the angle between $\vec{\mu}$ and $R$, which defines the $\mathrm{J} / \mathrm{H}$ aggregation. The energy splitting is zero when $\theta$ reaches $54.7^{\circ}$. While $\theta$ becomes larger than $54.7^{\circ}$, the allowed transition state blue shifts, dubbed as $\mathrm{H}$-aggregate. Conversely, the structure that makes the allowed state red-shifted is called J-aggregate, the emission of which becomes more red-shifted, corresponding to the prominent excimer emission. As for the simple case like $\mathbf{C F}_{3^{-}}$ MTTM, the relevant short intermolecular distance $4.68 \AA$ (center to center) and J-aggregation-like $\theta$ angle $46.7^{\circ}$ (Fig. 1e) lead to excimer formation. Note that despite similar $\mathrm{R}$ and $\theta$ between $\mathbf{C F}_{3}-\mathbf{M T T H}$ and $\mathbf{C F}_{3}-\mathbf{M T T M}, \mathbf{C F}_{3}-\mathbf{M T T H}$ with ESIPT reveals negligible excimer emission, which will be elaborated in the section of time-resolved measurements (see below). Moreover, We also make a fair comparison with the parent moiety HTTH, the reported compound $\boldsymbol{t}$-HTTH ${ }^{36}$ and the other derivatives $\boldsymbol{m}$ HTTH and F-HTTH (Supplementary Figure 5 and Supplementary Figure 6). Importantly, compounds HTTH, F-HTTH, mHTTH and $\boldsymbol{t}$-HTTH ${ }^{36}$ with $-\mathrm{H},-\mathrm{F},-\mathrm{CH}_{3}$, and $t$-butyl substituents all lack excimer emission (Supplementary Figure 7). The x-ray structure shows that the $\theta$ angles of F-HTTH are $86.5^{\circ}$, revealing an $\mathrm{H}$-aggregation-like structure. In spite of the $\mathrm{J}$ aggregate-like packing structures in other derivatives(Table 1), the long intermolecular distances $\mathrm{R}$ weaken the coupling of intermolecular interaction, suppressing the tendency of excimer formation.

As for $\mathbf{C F}_{3}-\mathbf{H T T H}$, owing to the lamellar packing structures, shown in Fig. 1e, the linear-polymer exciton model cannot directly apply to the case of $\mathbf{C F}_{3}-\mathbf{H T T H}$. To extend the model toward two dimensional aggregations, Hochstrasser et al. derived 

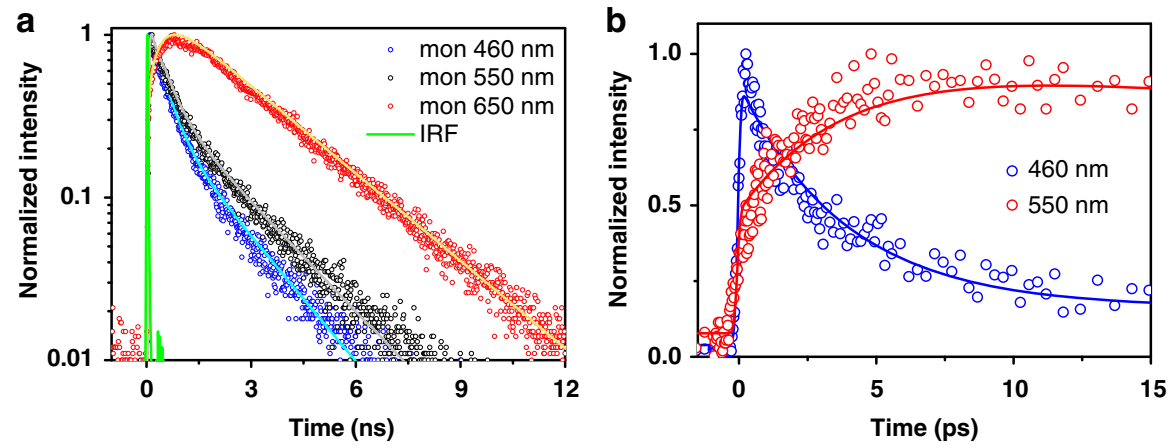

Fig. 2 Analyses of ESIPT coupled excimer reaction through time-resolved emission measurements. a Time-correlated single photon counting measurement of $\mathbf{C F}_{\mathbf{3}}-\mathbf{H T T H}$ in solid state of pristine powder at room temperature. $\mathbf{b}$ Fluorescence up-conversion decay curves of $\mathbf{C F}_{\mathbf{3}}-\mathbf{H} \mathbf{H T H}_{\mathbf{T}}$ in solid state of pristine powder at room temperature. The data were acquired at $\lambda_{\text {exc }}=400 \mathrm{~nm}$

Table 2 Steady-state and time-resolved photophysical properties of $\mathrm{CF}_{3}-\mathrm{HTTH}$ in solid state of pristine powder at room temperature

Steady-state measurement

\begin{tabular}{ll}
\hline$\lambda_{\text {em }}[\mathrm{nm}]$ & $\boldsymbol{\Phi}_{\boldsymbol{f}}$ \\
\hline $\mathrm{N}^{\star}: 460$ & 0.27
\end{tabular}

$T^{\star}: 550$

$E^{*}: 600$

Time-resolved measurement

\begin{tabular}{lllll}
\hline $\boldsymbol{\tau}_{\text {obs }}$ [ps] (pre-exp. factor) & \\
$3.9(0.85), 386(0.15), 1654(0.04)^{\mathrm{c}}$ & $\boldsymbol{\tau}_{\mathbf{p t}}$ [ps] & $\boldsymbol{\tau}_{\text {-pt }}$ [ps] & $\boldsymbol{K}_{\text {eq }}$ & $\boldsymbol{\Delta E}^{\star}\left[\mathbf{k c a l}_{\mathbf{~ m o l}}{ }^{\mathbf{1}}\right]^{\mathbf{b}}$ \\
$3.8(-0.37), 386(0.63), 1654(0.17)^{\mathrm{c}}$ & & 26.0 & 5.7 & -1.0 \\
$386(-0.41), 2430(0.59)^{\mathrm{c}}$ & & & &
\end{tabular}

normal emission, $T$ tautomer (ESIPT) emission, $E$ excimeric emission, $\tau_{\mathrm{pt}}$ proton transfer time constant, $\tau_{-\mathrm{pt}}$ reverse proton-transfer time constant, $K_{\mathrm{eq}}=\tau_{-p t} / \tau_{\mathrm{pt}}$, equilibrium constant

aObserved emission lifetimes $\left(\tau_{\mathrm{obs}}\right)$ by femtosecond fluorescence up-conversion

Energy differences $\left(\Delta \mathrm{E}^{\star}=E^{\star}\right.$ tautomer $\left.-E_{\text {normal }}^{\star}\right)$ between the normal form and tautomer form species in the lowest excited state $\left(S_{1}\right)$

CPopulation decay time constant was determined by subnanosecond time-correlated single photon counting system

the exciton coupling model in the lamellar system shown below: ${ }^{39}$

$$
\Delta E_{\text {lamellar }} \sim \frac{8|\vec{\mu}|^{2}}{4 \pi \varepsilon d^{3}}
$$

Intriguingly, the $\theta$-dependent term is canceled out by summing over the whole layer of transition dipoles, leaving the spatial term of interplanar distance $d$. This difference greatly enhances the exciton coupling of $\mathbf{C F}_{\mathbf{3}}-\mathbf{H T T H}$. Since the interplanar distance $\mathrm{d}$ is much shorter than the intermolecular distance $\mathrm{R}$ especially for J-aggregation-like structures, the coupling strength of $\mathbf{C F}_{\mathbf{3}}-\mathbf{H T T H}$ is expected to be larger than other derivatives, leading to the most feasible excimer formation among series congeners listed in Table 1. Empirically, it seems like that the prerequisite of excimer formation lies in the symmetric dual $\mathrm{OCH}_{3}$-thiazolo[5,4- $\left.d\right]$ or -OH-thiazolo[5,4-d] chromophore such as $\mathbf{C F}_{3}-\mathbf{H T T H}$ and $\mathbf{C F}_{\mathbf{3}}$ MTTM, which also requires $-\mathrm{CF}_{3}$ to occupy suitable space for adjusting layer-by-layer structure with small $\theta$. This perhaps is due to the weak polarizability and decent size of $-\mathrm{CF}_{3}$. Moreover, it has been reported that the interaction of $\mathrm{C}-\mathrm{F} \bullet \bullet \cdot \mathrm{H}-\mathrm{C}$ helps to align the molecules, which leads to different molecular organization from those without $-\mathrm{CF}_{3}$ group ${ }^{40,41}$.

Time-resolved measurements of CF3-HTTH in solid. We then performed time-resolved fluorescence measurements in attempts to shed light on the associated reaction kinetics. We utilized picoto nanosecond time-correlated single-photon counting system (TCSPC, system response time $\sim 20$ ps) to probe dynamics of relaxation of the multiple emission at room temperature. Upon exciting (400 nm) $\mathbf{C F}_{3}$-HTTH and monitoring at $650 \mathrm{~nm}$ where the excimer emission is predominant, a long rise component of
400 ps is obvious from TCSPC measurement (Fig. 2a), followed by a population decay of $2.4 \mathrm{~ns}$. This 400 ps time constant is also observed in the decay component of both normal and proton transfer tautomer emission monitored at $460 \mathrm{~nm}$ and $550 \mathrm{~nm}$ emission, respectively (Fig. 2a and Table 2), indicating that the dynamics of ESIPT and excimeric formation are mutually correlated (see below). In addition to the 400 ps decay component for both normal and tautomer emission bands, careful examination on Fig. 2 a indicates that there exists a minor $(\sim 10 \%)$ slow decay component of $1.7 \mathrm{~ns}$ when monitoring at either normal $(460 \mathrm{~nm})$ or tautomer $(550 \mathrm{~nm})$ emission band. This nonnegligible component did not decrease its ratio of contribution after several times recrystallization. Therefore, its origin from the impurity is eliminated. More plausibly, the $1.7 \mathrm{~ns}$ component originates from the surface effect of the solid. We will elaborate this in detail in the following section regarding the spatial distribution of the emission properties in the solid crystal by twophoton excitation emission microscopy (see below).

We then further probe the faster ESIPT dynamics of $\mathbf{C F}_{\mathbf{3}^{-}}$ HTTH, which cannot be resolved by TCSPC and has to be further resolved by femtosecond fluorescence up-conversion technique (system response time $\sim 120 \mathrm{fs}$ ). Figure $2 \mathrm{~b}$ clearly shows that the decay time of the normal emission $(460 \mathrm{~nm})$ of $3.9 \pm 0.2 \mathrm{ps}$, within experimental error, is identical with the rise time $(3.8 \pm 0.2 \mathrm{ps})$ of the tautomer, both of which are followed by a long population decay that is measured to be 400 ps by TCSPC. According to the coupling reaction kinetics ${ }^{31}$, mathematically, the ratio of preexponential value for short versus long decay components of $\mathrm{N}^{*}$ is equivalent to equilibrium constant $K_{\mathrm{eq}}$ between $\mathrm{N}^{*}$ and $\mathrm{T}^{\star}$, which is defined as $K_{e q}=k_{p t} / k_{-p t}$. This, together with $k_{p t}+k_{-p t}$ $=(3.9 \mathrm{ps})^{-1}, k_{\mathrm{pt}}$ and $k_{-\mathrm{pt}}$ are deduced to be $(4.6 \mathrm{ps})^{-1}$ and 

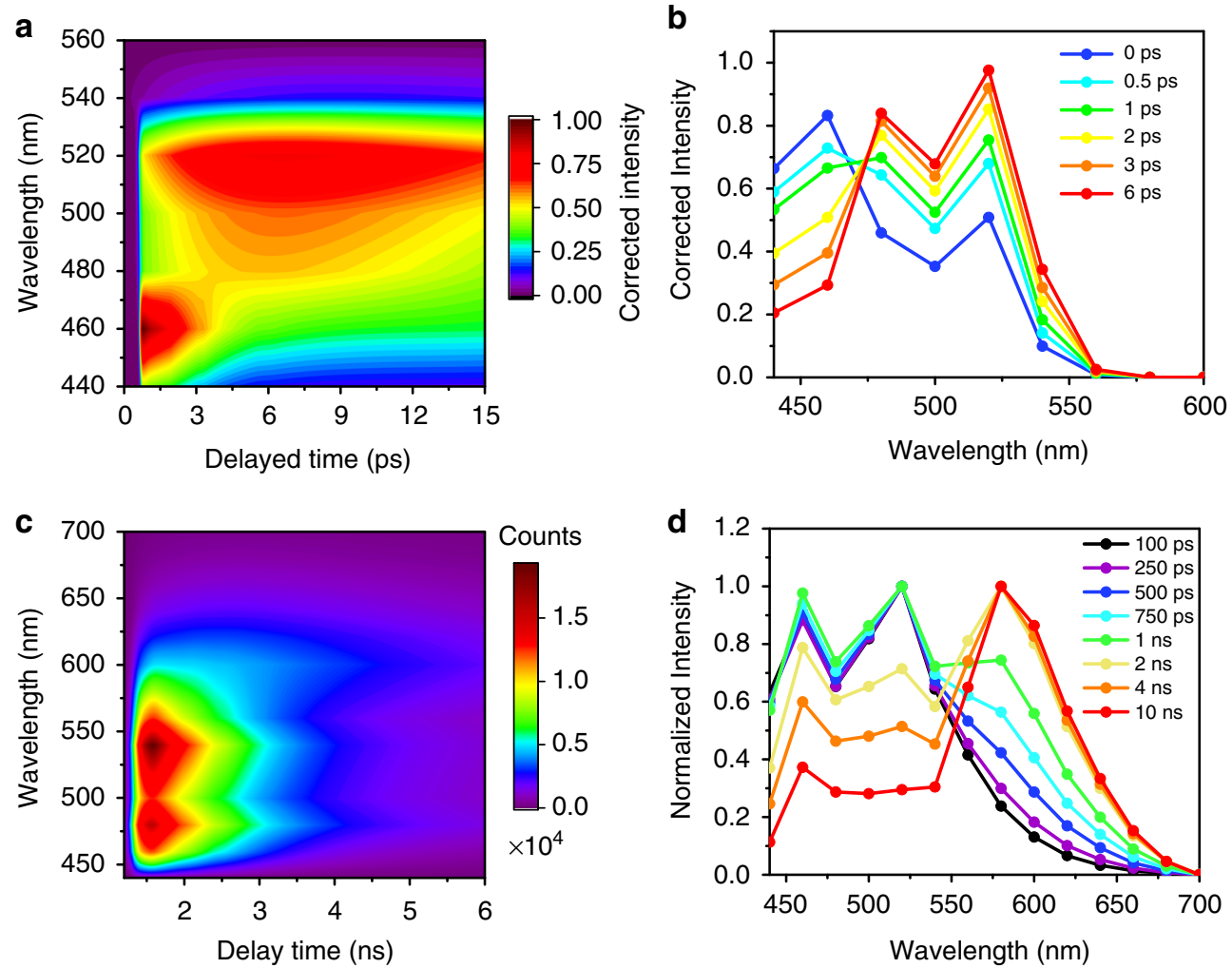

Fig. 3 Time-resolved photoluminescence spectra of $\mathbf{C F}_{\mathbf{3}}-\mathbf{H T T H}$ in pristine solid powder at room temperature. $\mathbf{a}, \mathbf{b}$ are femtosecond TRPL and the corresponding emission spectra at a time specified in the inset. Note that both peak intensities are calibrated by steady-state emission spectra in pristine powder shown in Fig. 1c. c, $\mathbf{d}$ are pico-nanosecond TRPL and the corresponding emission spectra at a time specified in the inset. $\mathbf{c}$ is the raw data without normalization and (d) is normalized at the intensity maximum for each delayed time specified in the inset. The data was acquired at $\lambda_{\text {exc }}=400 \mathrm{~nm}$

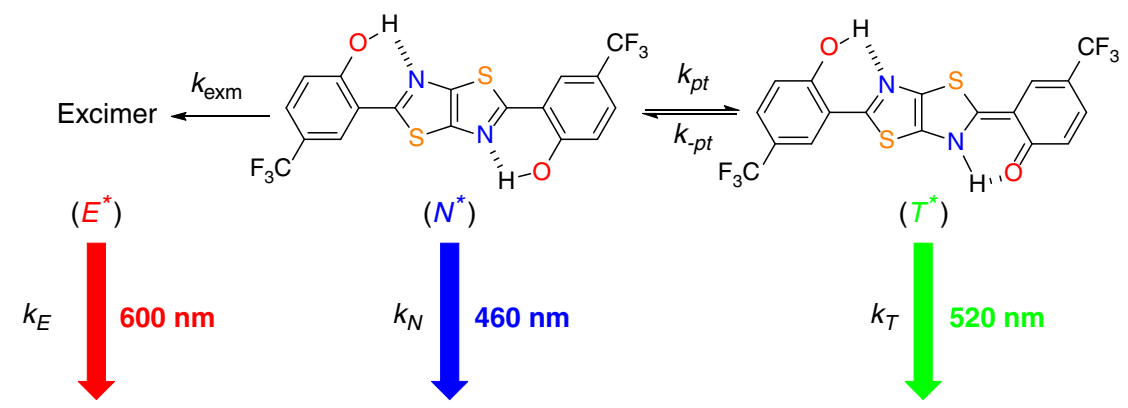

Fig. 4 The proposed kinetic model for ESIPT coupled excimer reaction of $\mathbf{C F}_{\mathbf{3}}-\mathbf{H} \mathbf{H T H}$ in the solid crystal. $k_{N}$ and $k_{T}$ are the population decay rate constants for the excited normal $\mathrm{N}^{\star}$ and tautomer $\mathrm{T}^{\star}$ states, respectively. $k_{p t}$ and $k_{-p t}$ are forward and reverse proton transfer rate constants, respectively. $k_{\text {exm }}$ is the rate constant of the excimer formation. $k_{E}$ is the population decay of the excimer $\mathrm{E}^{\star}$

$(26.0 \mathrm{ps})^{-1}$, respectively. In comparison to $\mathbf{C F}_{3}-\mathbf{H T T H}$, the larger $K_{\text {eq }}$ of $\mathbf{C F}_{3}$-MTTH (Table 2 and Supplementary Table 2) contributes to the more population of its tautomer, rendering dominant tautomeric emission and negligible excimeric emission.

To unravel the complex dynamics of $\mathbf{C F}_{3}-\mathbf{H T T H}$, we further perform time-resolved photoluminescence (TRPL) to visualize the temporal evolution of the emission. The results shown in Fig. 3 clearly indicate that at the normal excited state $\mathrm{N}^{*}$ populates to tautomeric state $\mathrm{T}^{*}$ correlating to the $3.9 \mathrm{ps}$ decay at $\sim 460 \mathrm{~nm}$ band $\left(\mathrm{N}^{*}\right)$ and the consonant rise of the $\sim 550 \mathrm{~nm}$ band $\left(\mathrm{T}^{*}\right)$. Afterwards, the system virtually reaches fastequilibrium within the first $10 \mathrm{ps}$ owing to the relatively slow dynamics of excimer formation (400 ps) (Fig. 3a, b). Subsequently, both $\mathrm{N}^{*}(460 \mathrm{~nm})$ and $\mathrm{T}^{*}(550 \mathrm{~nm})$ emissions decline, accompanied by the increase of the $600 \mathrm{~nm}$ excimer emission band $\left(E^{\star}\right)$ with a $\sim 400$ ps time constant, which then gradually decays within a time window of several nanoseconds. These results clearly demonstrate the correlations of relaxation dynamics among $\mathrm{N}^{*}, \mathrm{~T}^{*}$, and excimer $\mathrm{E}^{*}$.

Simulation of the kinetic model of $\mathrm{CF}_{3}$-HTTH solid. Standing on above dynamic data, we then propose a mechanism depicted in Fig. 4, which incorporates a simultaneous ESIPT coupled excimer reaction among $\mathrm{N}^{*}, \mathrm{~T}^{*}$ and $\mathrm{E}^{*}$. The feasibility of Fig. 4 is then rigorously tested by the time-evolution simulation. Fundamentally, excimer formation cannot be directly from the tautomer because none of ground-state tautomer is adjacent to the excited tautomer. In addition, because similar excimer formation is observed in $\mathbf{C F}_{3}-\mathbf{M T T M}$ where no ESIPT takes place, the possibility of exciplex formation via an excited tautomer and a ground-state normal species can be discarded as well. 

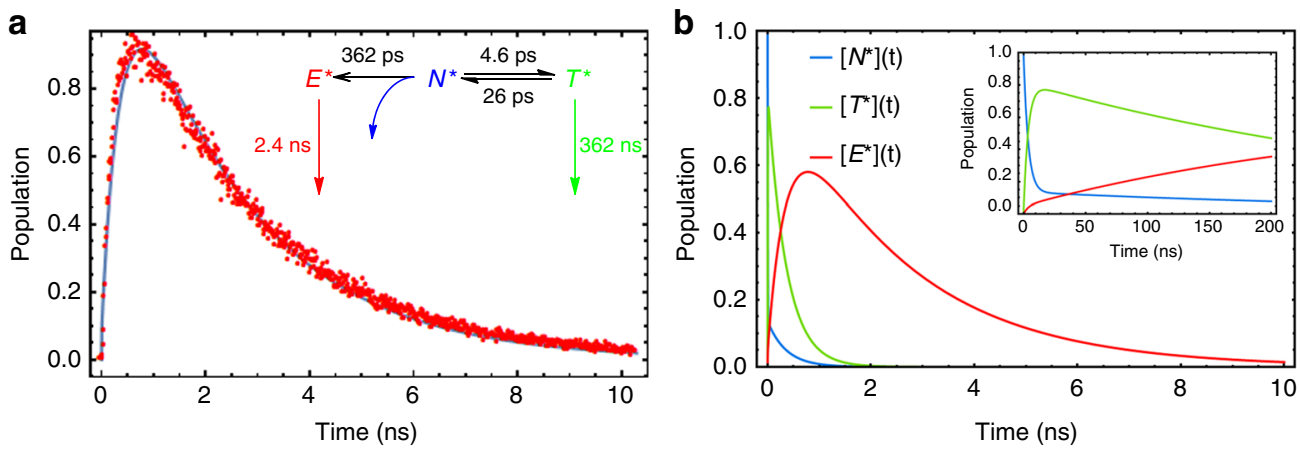

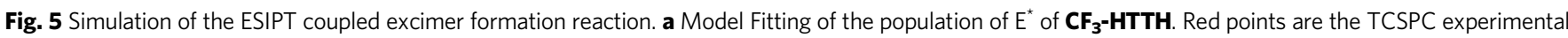
data of $\mathbf{C F}_{\mathbf{3}} \mathbf{- H T T H}$ in solid state monitored at $650 \mathrm{~nm}$. Blue line shows the calculated result of the evolution of the population of excimer. The inset is the proposed kinetic model for the ESIPT coupled excimer reaction and the best fitted parameters. Note that the time constants are taken from Eqs. 6-8, not the observed time constants. $\mathbf{b}$ Plot of the analytical solution of the time-evolution of the excited states of $\mathbf{C F}_{3}-\mathbf{H T T H}$ for $\mathrm{N}^{\star}$, $\mathrm{T}^{\star}$ and $\mathrm{E}^{\star}$ in the crystal kernel

Accordingly, the relaxation dynamics of each excited species can be expressed as follows.

$$
\begin{gathered}
\frac{\mathrm{d} N^{*}}{\mathrm{~d} t}=-\left(k_{N}+k_{\mathrm{pt}}+k_{\mathrm{exm}}\right) \times N^{*}(t)+k_{-\mathrm{pt}} \times T^{*}(t), \\
\frac{\mathrm{d} T^{*}}{\mathrm{~d} t}=-\left(k_{T}+k_{-\mathrm{pt}}\right) \cdot T^{*}(t)+k_{\mathrm{pt}} \cdot N^{*}(t), \\
\frac{\mathrm{d} E^{*}}{\mathrm{~d} t}=-k_{E} \cdot E^{*}(t)+k_{\mathrm{exm}} \cdot N^{*}(t) .
\end{gathered}
$$

We then performed a computer simulation based on the kinetic Eqs. (3-5) and obtained the best fit to the experimental data listed in Table 2. To carry out the simulation, we utilize the results of the time-resolved measurements as the initial values for CF $_{3}$-HTTH, for which $(4.6 \mathrm{ps})^{-1},(26.0 \mathrm{ps})^{-1},(400, \mathrm{ps})^{-1}$ and $(2.4 \mathrm{~ns})^{-1}$ are chosen for $k_{\mathrm{pt}}, k_{-\mathrm{pt}}, k_{\text {exm }}$ and $k_{E}$, while $k_{N}$, and $k_{T}$ are taken to be $(2.8 \mathrm{~ns})^{-1}$ from the values of $\mathbf{C F}_{\mathbf{3}}$-MTTH (Supplementary Table 2). We firstly attempt to fit the experimental curve obtained for $E^{*}$. This is simply because $E^{\star}$ does not involve the associated kinetics on the surface that may cause interference. After varying $k_{\text {exm }}, k_{N}$, and $k_{T}$ to perform the best fit to the timeevolution curve of $E^{*}$, the result is presented in Fig. 5a. The good match between the fitting curve and experimental results supports the validity of this approach. As a result, the best fitted kinetic parameters and the simulated time-resolved $\mathrm{N}(\mathrm{t})^{*}, \mathrm{~T}(\mathrm{t})^{*}$ and $\mathrm{E}(\mathrm{t})^{*}$ are shown in Eqs. (6-8) and the Fig. 5b, respectively, which match well with the experimental results above (Fig. 2a).

$$
\begin{gathered}
N^{*}(t)=0.87 \cdot e^{-t / 3.7 \mathrm{ps}}+0.13 \cdot e^{-t / 362 \mathrm{ps}} \\
T^{*}(t)=0.82 \cdot\left(e^{-t / 362 \mathrm{ps}}-e^{-t / 3.7 \mathrm{ps}}\right), \\
E^{*}(t)=0.95 \cdot e^{-t / 2.4 \mathrm{~ns}}-0.90 \cdot e^{-t / 362 \mathrm{ps}}-0.05 \cdot e^{-t / 3.7 \mathrm{ps}} .
\end{gathered}
$$

The forward $\left(k_{p t}\right)$ and reverse $\left(k_{-p t}\right)$ ESIPT are fitted to be $(4.6 \mathrm{ps})^{-1}$ and $(26 \mathrm{ps})^{-1}$, which together with other rate constants contribute to an overall early population decay time of 3.7 ps for $\mathrm{N}^{*}$ (Eqs. 12-15), which is in good match with the observed decay time of the $\mathrm{N}^{*}$ of 3.9 ps. Moreover, as shown in Fig. $5 \mathrm{~b}$, both $\mathrm{N}^{*}$ and $\mathrm{T}^{*}$ undergo a population decay time constant of 362 ps that correlates with the rise time of $E^{*}$ of 362 ps. These time constants are consonant with the corresponding $400 \mathrm{ps}$ time measured by TCSPC. Experimentally, the observed time constant of $400 \mathrm{ps}$ is the combination of $k_{N}, k_{T}, k_{\text {exm }}$, $k_{p t}$ and $k_{-p t}$ due to the reversibility of ESIPT reaction that links the population of $\mathrm{T}^{*}, \mathrm{~N}^{*}$ and $\mathrm{E}^{*}$ states together, leading to the same population decay lifetime of $\mathrm{T}^{*}$ and $\mathrm{N}^{*}$ state and the congruent rise lifetime of the $\mathrm{E}^{*}$ state. The irreversible excimer formation from the $\mathrm{N}^{*}$ state to the $\mathrm{E}^{*}$ state makes efficient population on $\mathrm{E}^{*}$, which then undergoes a population decay time of $2.4 \mathrm{~ns}$. This model proves that incorporation of reversible picosecond ESIPT and subnanosecond excimer formation dynamics is able to transfer the original population of $\mathrm{N}^{*}$ and $\mathrm{T}^{*}$ to $\mathrm{E}^{*}$ efficiently during the excited-state lifespan.

Morphology dependence and two-photon excitation microscopy. In addition to time-resolved information, we then meticulously inspect the morphology and spatial dependent emission spectra of $\mathbf{C F}_{3}-\mathbf{H T T H}$, in a form of pristine powder (used for the above measurements) and solid crystal. Importantly, the crystal of $\mathrm{CF}_{3}$-HTTH possesses dominant portion of the excimer emission. As we grind the crystal, the emission spectra become similar to that of pristine powder (Fig. 6a), demonstrating a mechanochromic property (Fig. 6b, c). Furthermore, as for the solid film of $\mathbf{C F}_{\mathbf{3}} \mathbf{- H T T H}$ prepared from the vapor deposition, no excimer can be observed (Supplementary Figure 4). We thus assert that the major cause of this phenomenon is the difference of morphology among solid film, powder and crystal, which mediates the formation of excimer for $\mathbf{C F}_{\mathbf{3}}$-HTTH. In solid film, on the one hand, $\mathbf{C F}_{3}$-HTTH molecules are more likely in random orientation, which lack suitable stacking arrangement and distance to proceed with excimer formation. On the other hand, $\mathbf{C F}_{\mathbf{3}}-\mathbf{H T T H}$ in powder has larger surface-to- volume ratio, in which the higher surface energy and fewer neighbor molecules (cf. crystal) inhibit the formation of excimer. As a result, both normal and tautomer emission are enhanced on the surface of the powder samples. What is more, by measuring the dynamics of film and grounded samples, we found that the contribution of emission of $1.7 \mathrm{~ns}$ decay in both powder and ground samples is greatly enhanced (Supplementary Table 3), revealing the close relationship between the dynamics of $1.7 \mathrm{~ns}$ decay component and surface properties.

We thus propose that the minor $1.7 \mathrm{~ns}$ components originate from the solid surface where mainly ESIPT takes place. To prove our hypothesis, we develop a methodology to study the luminescence and photodynamics at different depths of a crystal. Taking advantage of two-photon excitation microscopy, we are able to measure both steady-state emission spectrum and lifetime distribution from surface to kernel of the $\mathbf{C F}_{\mathbf{3}}-\mathbf{H T T H}$ crystal. The spatially resulted emission spectra, shown in Fig. $7 \mathrm{a}, \mathrm{b}$, indicate that $\mathbf{C F}_{\mathbf{3}^{-}}$ HTTH molecules on the surface are inclined to form normal and tautomeric states rather than generating excimer. As we focus the $800 \mathrm{~nm}$ two-photon excitation light beneath the surface by $\sim 6 \mu \mathrm{m}$, which is close to the kernel of the prepared crystal, the emission spectrum is majorly dominant by excimer instead of normal and 
a

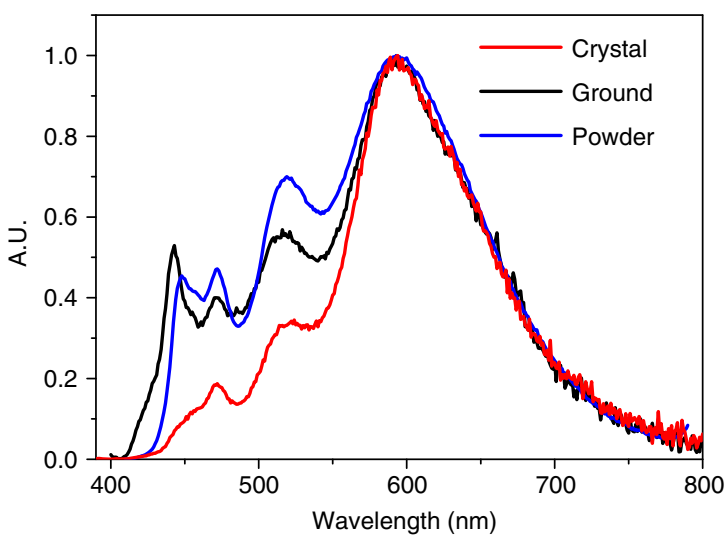

b
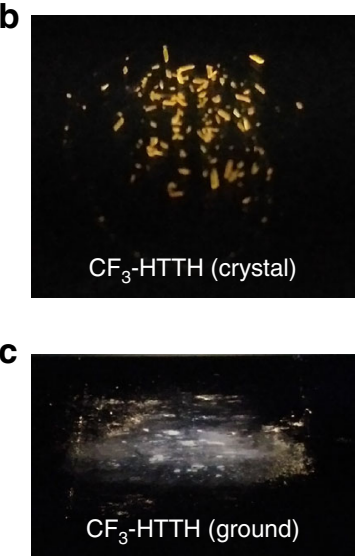

Fig. 6 Mechanochromism of $\mathbf{C F}_{\mathbf{3}}-\mathbf{H T T H}$. a Steady-state emission of $\mathbf{C F}_{\mathbf{3}}-\mathbf{H T T H}$ in pristine powder, solid crystal and the ground solid crystal at room temperature. The data was acquired at $\lambda_{\mathrm{exc}}=380 \mathrm{~nm}$. $\mathbf{b}, \mathbf{c}$ are the photos of the solid crystal and the ground solid crystal of $\mathbf{C F}_{\mathbf{3}}-\mathbf{H T T H}_{\mathbf{H}}$
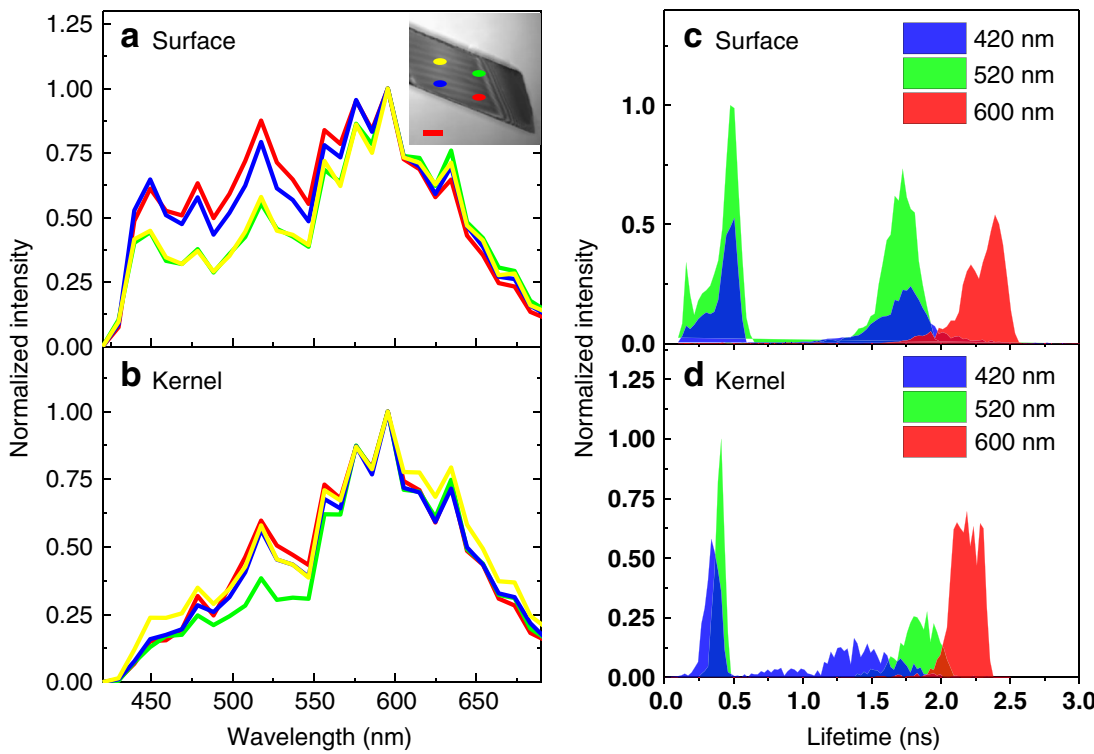

Fig. 7 Dependence of steady-state and time-resolved emission in different depths of crystal. $\mathbf{a}, \mathbf{b}$ are the steady-state emission spectra of the crystal of $\mathbf{C F}_{\mathbf{3}^{-}}$ HTTH at surface and kernel (beneath the surface for $6 \mu \mathrm{m}$ ) at different horizontal positions at room temperature respectively. The inset is the bright field image measured by $800 \mathrm{~nm}$ excitation with the 50-um red scale bar. Each color spot corresponds to the measured region of the emission spectra. The two-photon excitation wavelength is $800 \mathrm{~nm}$. $\mathbf{c}, \mathbf{d}$ are the lifetime distribution of molecules at crystal surface and kernel of $\mathbf{C F}_{\mathbf{3}}-\mathbf{H T T H}$ at room temperature respectively. Three exponential decay functions are utilized to fit the population lifetime at each emission wavelength. Blue, green, and red areas correspond to the decay lifetimes monitoring at 420,520,600 nm, representing normal, tautomer, and excimer emission, respectively. The excitation wavelength is $800 \mathrm{~nm}(\sim 100 \mathrm{fs}$ pulse)

tautomer emission (Fig. 7b). The difference between Fig. 7a, b reveals that surface of the crystal may disrupt the molecular packing and hence hinders the excimer formation, whereas ESIPT is intrinsic, and still takes place on the surface. If so, the emission relaxation dynamics between surface and kernel of the crystal are expected to be different, which should provide another crucial evidence. The lifetime distribution of $\mathbf{C F}_{\mathbf{3}}-\mathbf{H T T H}$ at crystal surface and kernel, shown in Fig. 7c, d, respectively, does exhibit depthdependent multiple relaxation dynamics. The $\sim 400$ ps of $420 \mathrm{~nm}$ and $520 \mathrm{~nm}$ and the $\sim 2.4 \mathrm{~ns}$ decay of $600 \mathrm{~nm}$ are assigned to normal, tautomeric, and excimeric population decay time constants, respectively which is consistent with time-resolved data listed in Table 2. Compared to that in kernel, crystal surface has significantly more and narrower lifetime distribution at 1.5-1.7 ns (within experimental fitting uncertainty), revealing that the emission population decay in this timescale majorly takes place on the surface. It is thus clear that the $1.7 \mathrm{~ns}$ decay component is assigned to both normal and tautomeric population decay where only equilibrium type ESIPT takes place on the surface ${ }^{31,36}$. To sum up, the mechanochromic effect in our system arises from surface and inner emission of crystal owing to distinct degree of packing, which leads to multiple decay lifetimes.

\section{Discussion}

In this work, using $\mathbf{C F}_{\mathbf{3}}$-HTTH as a prototype, we provide a mechanism to pinpoint the ESIPT coupled excimer reaction and the origin of mechanochromism. Steady-state emission spectra of $\mathbf{C F}_{3^{-}}$ HTTH in solid state show three distinct bands consisting of conventional, proton-transfer tautomer, and excimer emission covering the panchromatic $420 \mathrm{~nm}$ to $750 \mathrm{~nm}$ region. The ratiometric of these emission bands are strongly dependent on the solid morphology, as 
indicated by the dominant red excimer emission in crystal to the lack of excimer emission in the solid film, and powder in between. Timeresolved measurements, combined with simulations indicate a parallel kinetic model of ESIPT coupled excimer reaction. With the help of two-photon excitation microscopy, the spatially and time-resolved emission of the $\mathbf{C F}_{3}-\mathbf{H T T H}$ crystal leads us to conclude that the excimer formation is prohibited on the surface due to large surface energy and disfavored intermolecular orientation. Inside the crystal kernel, proper orientation of neighbor molecules facilitates the formation of excimer after fast equilibrium of ESIPT reaction.

We believe our system is not a sporadic case but may be generalized to other mechanochromic phenomena where multiple, irregular relaxation dynamics in solid have been frequently encountered $^{42-45}$. The mechanochromic emission is conventionally attributed to differentw $\pi-\pi$ and/or other intermolecular interactions induced by conformational change after applying mechanic force ${ }^{44,45}$, while the role of surface-to- volume effect is rarely studied. Owing to the rapid emergence of luminescence materials, it is timely as well as urgent to gain fundamental insights into the mechanism of photophysics in solids. Of particular interest is to probe the origin of peculiar luminescence spectra that are commonly encountered in solid. Unfortunately, the emission from the ensemble has received more attention and the distinction regarding luminescence properties between surface and kernel of organic solids is frequently ignored. In this study, we demonstrate the difference in photophysical properties between surface and kernel in solid crystal and anticipate that this new insight may start to elucidate the correlation among spatial dependent luminescent properties, mechanochromism, and aggregation-induced phenomena.

\section{Methods}

Synthesis and characterization. Dichloromethane $\left(\mathrm{CH}_{2} \mathrm{Cl}_{2}\right)$ and N,N-dimethylformamide (DMF) were distilled from appropriate drying agents prior to use. Commercially available reagents were directly used without further purification. Flash column chromatography was carried out using silica gel from Merck (230-400 mesh). Detailed synthesis are shown in Supplementary Methods. ${ }^{1} \mathrm{H}$ NMR, ${ }^{13} \mathrm{C}$ NMR and ${ }^{19} \mathrm{~F}$ NMR spectra were recorded on Varian Unity 400 at $400 \mathrm{MHz}, 100 \mathrm{MHz}$ and 376 $\mathrm{MHz}$, respectively. High resolution mass spectra were recorded by Gas

Chromatograph-Mass Spectrometer (Finnigan MAT TSQ-46C GC/MS/MS/DS). NMR spectra are available in Supplementary Figures 8-16. The X-ray diffraction intensity data were collected at $293 \mathrm{~K}, 140 \mathrm{~K}$ or $133 \mathrm{~K}$ on a Rigaku RAXIS RAPID IP imaging plate system with MoKa radiation $(\lambda=0.71073 \AA)$. The crystal data and structure refinement of the all compounds are provided in Supplementary Table 4-9.

\section{Steady-state and time-resolved spectropic measurements. Steady-state} absorption and emission spectra were recorded by a Hitachi (U-3310) spectrophotometer and an Edinburgh (FS920) fluorimeter, respectively. Detailed timeresolved spectroscopic measurements were reported previously elsewhere ${ }^{46}$. In brief, nanosecond time-resolved studies were performed by an Edinburgh FL 900 timecorrelated single photon-counting (TCSPC) system with a pulsed hydrogen-/or nitrogen-filled lamp as the excitation light source. The data were fitted with sum of exponential functions using the nonlinear least-squares procedure in combination with the convolution method. Subnanosecond to nanosecond time-resolved studies were used another TCSPC system (OB-900 L lifetime spectrometer, Edinburgh). The excitation light source from the second-harmonic generation ( $\mathrm{SHG}, 380 \mathrm{~nm}$ ) of pulse selected femtosecond laser pulses at $760 \mathrm{~nm}$ (Tsunami, Spectra-Physics). The fluorescence was collected at a right angle with respect to the pump beam path and passed through a polarizer, which setting the polarization at magic angle $\left(54.7^{\circ}\right)$ to eliminate anisotropy. Similar data analysis and fitting procedures were applied. The temporal resolution, after partial removal of the instrumental time broadening, was $\sim 20$ ps. The femtosecond fluorescence up-conversion (FOG100, CDP) was utilized to study ultrafast dynamics of the titled compounds. The excitation light source was generated from from the second-harmonic generation (SHG, $380 \mathrm{~nm}$ ) of pulse selected femtosecond laser pulses at $760 \mathrm{~nm}$. The cross correlation (instrument response function) obtained from the Raman scattering signal showed a full width at half-maximum (FWHM) of $\sim 150 \mathrm{fs}$ and therefore a temporal resolution of $\sim 120 \mathrm{fs}$ after deconvolution could be obtained. The polarization of the excitation laser pulses was set parallel, vertical, or at the magic angle $\left(54.7^{\circ}\right)$ by a $\lambda / 2$ waveplate with respect to the detection polarization direction for ultrafast anisotropic measurement or anisotropy-free fluorescence decay. On the other hand, we used Zeiss LSM 710 (Zeiss, Germany) equipped with $80 \mathrm{MHz}$ fs mode-locked Ti:sapphire laser (Mai-Tai DeepSee, Spectra-Physics) for the two-photon excitation microscopy. To collect signals, we used Plan-Apochromat $63 \times / 1.4$ oil immersion objective lens
(Zeiss, Germany). Single-photon counting board SPC-150, 16-channel spectral detector PML-16-1-C and programmable pulse generator DDG-210 (Becker\&Hickl $\mathrm{GmbH}$, Germany) were utilized to measure lifetime distribution of crystal. The DDG-210 is triggered by the pixel clock of the LSM 710 and de-livers a laser modulation signal of programmable width, which is fed back into the beam blanking system of the microscope. The TCSPC data were analyzed by the SPCImage 6.0 software (Becker \& Hickl GmbH, Germany).

Kinetic simulation of $\mathbf{C F}_{\mathbf{3}}-\mathbf{H T T H}$ in solid state. The simulation is performed by Wolfram Mathematica 10.1. We choose the experimental data of $\mathbf{C F}_{\mathbf{3}}-\mathbf{H T T H}$ in solid state of pristine powder from the time-correlated single photon counting (TCSPC) system (OB-900 L lifetime spectrometer, Edinburgh) with the excitation at $400 \mathrm{~nm}$ and monitored at $650 \mathrm{~nm}$. We assume the kinetic model is followed by Scheme 2. The rate equations of this model can be expressed as follows by Eqs. (24). These equations can be numerically solved by given initial condition $\left[\mathrm{N}^{*}\right](\mathrm{t})=1$, $\left[\mathrm{T}^{*}\right](\mathrm{t})=0,\left[\mathrm{E}^{*}\right](\mathrm{t})=0$ at $\mathrm{t}=0$ and with fixed parameters: $k_{p t}, k_{-p t}$ and $k_{E}$ equal to $(4.6 \mathrm{ps})^{-1},(26.0 \mathrm{ps})^{-1},(400, \mathrm{ps})^{-1}$ and $(2.4 \mathrm{~ns})^{-1}$ respectively $\mathrm{k}_{\mathrm{pt}}=2 \times 10^{12} \mathrm{sec}^{-1}$ $\mathrm{k}_{\mathrm{pt}}=2 \times 10^{12} \mathrm{sec}^{-1}$. We made the left parameters $\left(k_{\text {exm }}, k_{N}\right.$, amplitude) be free to fit the raw data with chosen $k_{T}$ taken to be $(2.8 \mathrm{~ns})^{-1}$ from the values of $\mathbf{C F}_{3}-\mathbf{M T T H}$ (Supplementary Table 2). As we get the rate constants $k_{\text {exm }}$ and $k_{N}$, we fix all rate constants and keep the same initial value to solve the coupled equations analytically, which shows the population evolution of $\mathrm{N}^{*}$ and $\mathrm{T}^{*}$ (Fig. 4). On the other hand, the symbolic representation shows the origin of the observed lifetime exhibiting coupling reaction of ESIPT and excimer formation (Eqs. 9-15).

$$
\begin{gathered}
N^{*}(t)=A_{1} \cdot e^{-\lambda_{1} t}+A_{2} \cdot e^{-\lambda_{2} t} \\
T^{*}(t)=B \cdot\left(e^{-\lambda_{1} t}-e^{-\lambda_{2} t}\right) \\
E^{*}(t)=\mathrm{C}_{1} \cdot e^{-\lambda_{1} t}-C_{2} \cdot e^{-\lambda_{2} t}-C_{3} \cdot e^{-\lambda_{3} t} \\
\lambda_{1}=\frac{1}{2}\left(k_{\mathrm{tot}}-\sqrt{k_{\mathrm{tot}}^{2}-4\left(k_{T}+k_{-\mathrm{pt}}\right)\left(k_{N}+k_{\mathrm{exm}}\right)+k_{\mathrm{pt}} k_{T}}\right) \\
\lambda_{2}\left(k_{\mathrm{tot}}+\sqrt{k_{\mathrm{tot}}^{2}-4\left(k_{T}+k_{-p t}\right)\left(k_{N}+k_{\mathrm{exm}}\right)+k_{\mathrm{pt}} k_{T}}\right) \\
k_{\mathrm{tot}}=k_{N}+k_{\mathrm{exm}}+k_{T}+k_{-\mathrm{pt}}+k_{\mathrm{pt}} \cdot
\end{gathered}
$$

\section{Data Availability}

The data that support the findings of this study are available from the corresponding author on reasonable request. The X-ray crystallographic coordinates for the compounds reported in this article has been deposited at the Cambridge Crystallographic Data Centre (CCDC) under deposition number CCDC 1864267

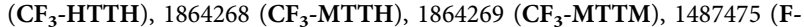
HTTH), 1062845 (HTTH), 1864270 ( $\boldsymbol{m}$-HTTH). These data can be obtained free of charge from The Cambridge Crystallographic Data Centre via www.ccdc.cam.ac. uk/data_request/cif.

Received: 15 October 2018 Accepted: 9 January 2019 Published online: 28 January 2019

\section{References}

1. Coe, S., Woo, W. K., Bawendi, M. \& Bulovic, V. Electroluminescence from single monolayers of nanocrystals in molecular organic devices. Nature 420, 800-803 (2002)

2. Mutai, T., Satou, H. \& Araki, K. Reproducible on-off switching of solid-state luminescence by controlling molecular packing through heat-mode interconversion. Nat. Mater. 4, 685-687 (2005).

3. Yan, D. P. et al. A cocrystal strategy to tune the luminescent properties of stilbene-type organic solid-state materials. Angew. Chem. Int. Ed. 50, 12483-12486 (2011)

4. Czerwieniec, R., Yu, J. B. \& Yersin, H. Blue-light emission of cu(i) complexes and singlet harvesting. Inorg. Chem. 50, 8293-8301 (2011).

5. Lee, J. et al. Deep blue phosphorescent organic light-emitting diodes with very high brightness and efficiency. Nat. Mater. 15, 92-98 (2016).

6. Miwa, T. et al. Blue organic light-emitting diodes realizing external quantum efficiency over $25 \%$ using thermally activated delayed fluorescence emitters. Sci. Rep. 7, 284 (2017). 
7. Qin, W. et al. Construction of efficient deep blue aggregation-induced emission luminogen from triphenylethene for nondoped organic lightemitting diodes. Chem. Mater. 27, 3892-3901 (2015).

8. Ravindran, E., Ananthakrishnan, S. J., Varathan, E., Subramanian, V. \& Somanathan, N. White light emitting single polymer from aggregation enhanced emission: a strategy through supramolecular assembly. J. Mater. Chem. C. 3, 4359-4371 (2015).

9. Xie, Z. L. et al. White-light emission strategy of a single organic compound with aggregation-induced emission and delayed fluorescence properties. Angew. Chem. Int. Ed. 54, 7181-7184 (2015).

10. Kim, D. H. et al. High-efficiency electroluminescence and amplified spontaneous emission from a thermally activated delayed fluorescent nearinfrared emitter. Nat. Photonics 12, 98-104 (2018).

11. Ly, K. T. et al. Near-infrared organic light-emitting diodes with very high external quantum efficiency and radiance. Nat. Photonics 11, 63-68 (2017).

12. Chen, L. et al. Ultrafast water sensing and thermal imaging by a metal-organic framework with switchable luminescence. Nat. Commun. 8, 15985 (2017).

13. Hofbeck, T. et al. Thermally Tunable Dual Emission of the d(8)-d(8) Dimer [Pt-2(mu-P2O5(BF2)(2))(4)](4-). Inorg. Chem. 55, 2441-2449 (2016).

14. Benito, Q. et al. Polymorphic copper iodide clusters: insights into the mechanochromic luminescence properties. J. Am. Chem. Soc. 136, 11311-11320 (2014).

15. Nakayama, H., Nishida, J., Takada, N., Sato, H. \& Yamashita, Y. Crystal structures and triboluminescence based on trifluoromethyl and pentafluorosulfanyl substituted asymmetric n-phenyl imide compounds. Chem. Mater. 24, 671-676 (2012).

16. Vahala, K. J. Optical microcavities. Nature 424, 839-846 (2003).

17. Wiersig, J. et al. Direct observation of correlations between individual photon emission events of a microcavity laser. Nature 460, 245-249 (2009).

18. Walther, C., Scalari, G., Amanti, M. I., Beck, M. \& Faist, J. Microcavity Laser Oscillating in a Circuit-Based Resonator. Science 327, 1495-1497 (2010).

19. Verhoeven, J. W. Glossary of terms used in photochemistry. Pure Appl. Chem. 68, 2223-2286 (1996).

20. Turro, N. J., Ramamurthy, V. \& Scaiano, J. C. Modern Molecular Photochemistry of Organic Molecules.. (University Science Books, Sausalito, Calif., 2010).

21. Khan, A. U. \& Kasha, M. Mechanism of 4-level laser action in solution excimer and excited-state proton-transfer cases. Proc. Natl. Acad. Sci. USA 80, $1767-1770$ (1983).

22. Stevens, B. Some Effects of molecular orientation on fluorescence emission and energy transfer in crystalline aromatic hydrocarbons. Spectrochim. Acta 18, 439-448 (1962).

23. Chen, C. L., Chen, Y. T., Demchenko, A. P. \& Chou, P. T. Amino proton donors in excited-state intramolecular proton-transfer reactions. Nat. Rev. Chem. 2, 131-143 (2018)

24. Demchenko, A. P., Tang, K. C. \& Chou, P. T. Excited-state proton coupled charge transfer modulated by molecular structure and media polarization. Chem. Soc. Rev. 42, 1379-1408 (2013).

25. Demchenko, A. P., Tomin, V. I. \& Chou, P. T. Breaking the kasha rule for more efficient photochemistry. Chem. Rev. 117, 13353-13381 (2017).

26. Li, G. Y. \& Han, K. L. The sensing mechanism studies of the fluorescent probes with electronically excited state calculations. Wiley Interdiscip. Rev. Comput. Mol. Sci. 8, e1351 (2018).

27. Zhao, G. J. \& Han, K. L. Hydrogen bonding in the electronic excited state. Acc. Chem. Res. 45, 404-413 (2012).

28. Zhou, P. W. \& Han, K. Unraveling the detailed mechanism of excited-state proton transfer. Acc. Chem. Res. 51, 1681-1690 (2018)

29. Goswami, S. et al. A chemodosimeter for the ratiometric detection of hydrazine based on return of esipt and its application in live-cell imaging. Org. Lett. 15, 5412-5415 (2013).

30. Park, S. et al. Imidazole-based excited-state intramolecular proton-transfer materials: Synthesis and amplified spontaneous emission from a large single crystal. J. Am. Chem. Soc. 127, 10070-10074 (2005).

31. Tang, K. C. et al. Fine tuning the energetics of excited-state intramolecular proton transfer (esipt): white light generation in a single esipt system. J. Am. Chem. Soc. 133, 17738-17745 (2011)

32. Mamada, M. et al. Highly efficient thermally activated delayed fluorescence from an excited-state intramolecular proton transfer System. ACS Cent. Sci. 3, 769-777 (2017).

33. Denk, W., Strickler, J. H. \& Webb, W. W. 2-photon laser scanning fluorescence microscopy. Science 248, 73-76 (1990).

34. So, P. T. C., Dong, C. Y., Masters, B. R. \& Berland, K. M. Two-photon excitation fluorescence microscopy. Annu. Rev. Biomed. Eng. 2, 399-429 (2000)

35. Samanta, S., Manna, U. \& Das, G. White-light emission from simple AIE-ESIPTexcimer tripled single molecular system. New J. Chem. 41, 1064-1072 (2017).
36. Zhang, Z. Y. et al. Control of the reversibility of excited-state intramolecular proton transfer (esipt) reaction: host-polarity tuning white organic light emitting diode on a new thiazolo[5,4-d]thiazole ESIPT system. Chem. Mater 28, 8815-8824 (2016)

37. Kasha, M., Rawls, H. R. \& Ashraf El-Bayoumi, M. The exciton model in molecular spectroscopy. Pure Appl. Chem. 11, 371 (1965).

38. McRae, E. G. \& Kasha, M. in The Molecular Exciton Model Physical Processes in Radiation Biology (ed. Leroy Augenstein, Ronald Mason, Barnett Rosenberg) 23-42 (Academic Press, 1964)

39. Hochstrasser, R. M. \& Kasha, M. Application of the exciton model to monomolecular lamellar systems. Photochem. Photobiol. 3, 317-331 (1964).

40. Shimoni, L., Carrell, H. L., Glusker, J. P. \& Coombs, M. M. Intermolecular effects in crystals of 11-(Trifluoromethyl)-15,16-dihydrocyclopenta[a] phenanthren-17-one. J. Am. Chem. Soc. 116, 8162-8168 (1994).

41. Thalladi, V. R. et al. C-H.F interactions in the crystal structures of some fluorobenzenes. J. Am. Chem. Soc. 120, 8702-8710 (1998).

42. Fang, M. M. et al. Triphenylamine derivatives: different molecular packing and the corresponding mechanoluminescent or mechanochromism property. J. Mater. Chem. C. 5, 9879-9885 (2017).

43. Gong, Y. Y. et al. Achieving persistent room temperature phosphorescence and remarkable mechanochromism from pure organic luminogens. Adv. Mater. 27, 6195-6201 (2015).

44. Mastropietro, T. F. et al. Luminescence mechanochromism in cyclometallated $\operatorname{Ir}(\mathrm{III})$ complexes containing picolylamine. Dalton Trans. 41, 8899-8907 (2012).

45. Mo, S. Z. et al. Tunable mechanoresponsive self-assembly of an amide-linked dyad with dual sensitivity of photochromism and mechanochromism. Adv. Funct. Mater. 27, 1701210 (2017)

46. Chou, P. T. et al. Femtosecond dynamics on excited-state proton chargetransfer reaction in 4'-N,N-Diethylamino-3-hydroxyflavone. The role of dipolar vectors in constructing a rational mechanism. J. Phys. Chem. A 109, 3777-3787 (2005)

\section{Acknowledgements}

We thank the ministry of science and technology (MOST 107-2628-M-002-017), Taiwan, for generous supports.

\section{Author contributions}

Y-C.W. carried out the dynamic simulation, analyzed and interpreted the photophysics and prepared the manuscript. Z.Z., S.-Y. H, and J.-C. L conducted the synthesis, purification, characterization. Y.-A.C. did the TCSPC and up-conversion measurements C.-H. W. carried out the two-photon excitation microscopic measurements. Z-Y.L conducted the TRPL measurements and analysis of data. J.-A.L. prepared the manuscript. P-T.C. developed the experimental approach, analyzed and interpreted the photophysics and prepared the manuscript. All authors discussed the results and contributed to the paper.

\section{Additional information}

Supplementary information accompanies this paper at https://doi.org/10.1038/s42004019-0113-8.

Competing interests: The authors declare no competing interests.

Reprints and permission information is available online at http://npg.nature.com/ reprintsandpermissions/

Publisher's note: Springer Nature remains neutral with regard to jurisdictional claims in published maps and institutional affiliations.

Open Access This article is licensed under a Creative Commons Attribution 4.0 International License, which permits use, sharing, adaptation, distribution and reproduction in any medium or format, as long as you give appropriate credit to the original author(s) and the source, provide a link to the Creative Commons license, and indicate if changes were made. The images or other third party material in this article are included in the article's Creative Commons license, unless indicated otherwise in a credit line to the material. If material is not included in the article's Creative Commons license and your intended use is not permitted by statutory regulation or exceeds the permitted use, you will need to obtain permission directly from the copyright holder. To view a copy of this license, visit http://creativecommons.org/ licenses/by/4.0/.

(C) The Author(s) 2019 